\title{
LIMITATIONS OF OBSERVATIONAL COSMOLOGY
}

\author{
MENAS KAFATOS \\ Department of Physics, George Mason University, Fairfax, VA 22030
}

\begin{abstract}
Unlike the usual situation with theoretical physics which is testable in the laboratory, in cosmological theories of the universe one faces the following problems: The observer is part of the system, the universe, and this system cannot be altered to test physical theory. Even though one can in principle consider any part of the observable universe as separate from the acts of observation, the very hypothesis of big bang implies that in the distant past, space-time regions containing current observers were part of the same system. One, therefore, faces a situation where the observer has to be considered as inherently a part of the entire system. The existence of horizons of knowledge in any cosmological view of the universe is then tantamount to inherent observational limits imposed by acts of observation and theory itself. For example, in the big bang cosmology the universe becomes opaque to radiation early on, and the images of extended distant galaxies merge for redshifts, $z$, of the order of a few. Moreover, in order to measure the distance of a remote galaxy to test any cosmological theory, one has to disperse its light to form a spectrum which would cause confusion with other background galaxies. Since the early universe should be described in quantum terms, it follows that the same problems regarding quantum reality and the role of the observer apply to the universe as a whole. One of the most fundamental properties of quantum theory, non-locality, may then apply equally well to the universe. Some of the problems facing big bang cosmology, like the horizon and flatness problems, may not then be preconditions on theoretical models but may instead be the manifestations of the quantum nature of the universe.
\end{abstract}

\section{Introduction}

With the theoretical framework of general relativity in place (Kafatos, 1989), Lemaitre and Friedmann postulated in the early 20's a dynamic, expanding and evolving universe. The Friedmann models obeyed the cosmological principle, which states that the universe is isotropic - the same in all directions - and homogeneous - of equal density, on the average, everywhere. On the other hand, to accommodate the obvious observational picture of Hubbles' expanding universe with a framework of an eternal universe, Herman Bondi, Thomas Gold and Fred Hoyle proposed in the late 40 's the steady state theory, describing a universe which although expanding would obey the perfect cosmological principle: the universe appears the same to all observers at all times.

In the early 50's George Gamow extended Lemaitre's and Friedmann's original ideas. Nuclear physics had progressed as a branch of physics and cosmologists could now use it to answer what might have happened in the early lifetime of the universe when it achieved energies and temperatures appropriate to nuclear physics. Soon observational astronomy provided strong evidence in favor of the big bang model, the existence of the 3 black body radiation and the existence of quarsars. The $3 \mathrm{~K}$ black body radiation consists of a microwave background of radiation that fills all

Y. Kondo (ed.), Observatories in Earth Orbit and Beyond, 543-550.

(C) 1990 Kluwer Academic Publishers. Printed in The Netherlands. 
space. In the big bang picture, this microwave background is the relic radiation from the initial big bang. It was predicted by Gamow, more than 10 years before it was accidentally discovered by Bell Telephone physicists Penzias and Wilson. The steady state theory cannot easily account for the nature of the background. The microwave radiation, it was believed, is a natural consequence of the big bang theory and no extra assumptions are needed. The second cosmological observation (Berry, 1976) that challenges steady state is the existence of the quasars. Discovered accidentally in the early sixties these objects appear to be very distant. In relativistic cosmology, as the universe expands, galaxies recede from each other and light from a distant galaxy would then be redshifted more to the red part of the spectrum than a nearby galaxy. Quasars have their spectral lines shifted so much to lower frequencies that must be at the edges of the observable universe, some of them receding away from us at speeds exceeding $90 \%$ the speed of light. According to big bang theory, at the billions of light years that a quasar is located, the galaxy is so faint that it cannot be observed. Only the brilliant star-like nucleus can be seen. These bright nuclei were very brilliant in the past compared to now, indicating that sources evolved as time went on. If this interpretation is correct, quasars violate the perfect cosmological principle because the universe does not look the same at all times - galaxies were much brighter in the past than today. Recently, though, it has become obvious that the big bang theory itself faces theoretical challenges not known in the early sixties. Yet, the vast majority of astronomers, cosmologists, and particle physicists still adhere to the big bang theory.

In the first phase after the big bang singularity the space-time description breaks down entirely, and this is followed by the so-called "inflationary era" at about $10^{-35} \mathrm{sec}$ (Guth and Steinhardt, 1984). By the time the universe had undergone inflation, it had expanded in size by a staggering factor of $10^{50}$ or more. When the expanding universe had cooled down to a temperature of about $10^{27} \mathrm{~K}$, the universe underwent a phase transition from the false vacuum where all the Higgs fields were zero to a less energetic phase which is the true vacuum of quantum theory (Barrow, 1988).

The inflationary model was originally proposed not because of a compelling theoretical reason but to solve some observational problems faced by the standard big bang theory of the universe. For example, the horizon of the universe, within which parts of the expanding primordial matter were in contact among themselves, expanded to a huge size becoming much larger than the rradius of the observable universe. This fact is critical to resolve the observational problems of the standard big bang without inflation. Unfortunately the details of the inflationary scenario are not uniquely determined today and can only presumably become known once the quantum field theory of unification of strong, electromagnetic and weak interactions - the GUT - is firmly in place.

\section{Observational Constraints of Cosmological Theory}

The original big bang theory of the universe could not account for a number of features revealed by observational cosmology. One is the observed curious property of the universe that the ratio of the observed density of matter in the universe is 
very close to the closure value i.e. $\Omega \simeq 1$ where $\Omega$ is the density ratio $\Omega=\rho / \rho_{\text {crit }}$ and (Barrow, 1984)

$$
\rho_{\text {crit }}=5 \times 10^{-30}\left(H_{0} / 50 \mathrm{~km} / \mathrm{sec} / \mathrm{Mpc}\right)^{2} \mathrm{gr} \mathrm{cm}^{-3}
$$

where $H_{0}$ is the Hubble constant. Were $\Omega$ turned out to be precisely equal to unity the geometry of the universe would be exactly flat and it would expand forever.

Current observations cannot unequivocally distinguish the type of the universe we live in. Values of for luminous matter are in the range 0.1 to 2 although most observers favor values close to 0.1 . If the only type of matter that there is in the universe is luminous matter found in stars and nebulae, this result would indicate an open universe. Even though present observations only indicate an approximate range of the mean density of the universe, this range is so close to the value of the critical density required for a flat geometry that many astronomers assume as a working hypothesis that the universe is exactly flat. If it turns out that the universe is not quite flat today, going back to the early times the universe must have been incredibly flat (Schramm, 1983) to one part in $10^{50}$. This coincidence is known as the flatness problem and indicates an incredible fine tuning in the initial conditions of the standard big bang universe.

The second problem facing big bang cosmology has to do with the uniformity of the $3 \mathrm{~K}$ black body radiation. Recent COBE observations indicate a remarkable uniformity of this radiation at all parts of the sky and an almost perfect correspondence with a black body radiation at $2.735 \mathrm{~K}$. In the hot big bang, though, opposite parts in the sky at the time that the microwave background formed 105 years from the beginning, were separated by distances of 107 light years (Schramm, 1983). Given the identity of temperatures from all parts of the sky and presuming that classical physics holds, one would conclude that opposite parts of the sky had to be in causal contact. This is known as the horizon problem. Again, it represents a problem of incredibly fine tuning in the conditions prevailing in the early universe or else a violation of classical causality.

Quantitative calculations show that slight anisotropies would not die away but on the contrary would get amplified. This is known as the isotropy problem. The problem becomes more severe in the steady state scenario which requires that whatever fluctuations in isotropy exist should be present at all levels. To be sure whether the universe turns out to be isotropic and homogeneous is an observational question. As we saw, the microwave radiation is highly isotropic. The universe is presumed to be expanding the same way in all directions, i.e. to be isotropic in matter as it is in background radiation. Moreover, the distribution of matter is presumed to reach homogeneity beyond the largest structures seen in the universe - the superclusters - i.e. beyond hundreds of millions of light years.

Recent observations challenge both these pillars of tradtional cosmological thinking: The universe may not be homogeneous, larger and larger structures have been found as the astronomer looks further and further into space (e.g. Burns et al., 1988). Galaxies seemingly cluster themselves to increasing hierarchies of clusters, superclusters and maybe even super-superclusters. Often these structures assume the form of filaments on the surface of very large bubbles with large "voids" in 
between. The universe may also not be isotropic in the motion of matter, galaxies have been found which do not move in the isotropic fashion of following the Hubble flow (Physics Today, 1989). Yet, the interesting question remains why the universe is not even more anisotropic than observed.

Taking all the evidence at face value, one would conclude that the universe requires incredibly fine tuning at the beginning, i.e. the universe represents a very unlikely "accident". Paul A.M. Dirac (1937) first noticed in the 30's that certain ratios involving fundamental constants of nature and physical parameters which at face value should not be related to them obey simple numerical relations. Some of these coincidences yield very large numbers which are not random as one might have expected, e.g.

$$
\begin{aligned}
& \text { Specific entropy } \simeq \frac{\text { number of photons }}{\text { number of baryons }} \simeq 10^{10} \\
& \frac{\text { Size of elementary particle }}{\text { Planck length }} \simeq 10^{20} \\
& \frac{\text { Electric force }}{\text { Gravitational force }} \simeq 10^{40} \\
& \frac{\text { Radius of observable universe }}{\text { Size of elementary particle }} \simeq 10^{40}
\end{aligned}
$$

Why the last, seemingly unrelated ratio, should be similar in value, which is a very large number, remains one of the most fundamental theoretical challenges facing physics today. Dirac believed this could not be a coincidence and formulated his large number hypothesis. Simply put, he reasoned that since as the universe expands its radius changes in value, in order for the ratios to be equal today one of the quantitites in the third ratio also has to change in time. He postulated that Newton's gravitational constant changes in time. Attempts to verify Dirac's hypothesis have so far failed.

Today some physicists have postulated that our existence as observers requires this fine tuning and that the seemingly unrelated ratios numerically giving such improbable values point to our existence as necessitating the kind of universe we live in. Put differently, the universe is unique because it contains conscious observers. This is known as the anthropic principle (Barrow and Tipler, 1986). Various flavors of anthropic principles exist, some of them even requiring a specific future evolution in order to preserve the transfer of information between observers.

\section{Horizons of Knowledge in Cosmology}

As we study the observations pertaining to the early universe, we encounter a number of observational horizons of knowledge (Kafatos, 1989). These observational horizons have to do with the quantum nature of light. For example, attempting to obtain the distance of a faint galaxy requires that we obtain its spectrum. To obtain an accurate spectrum requires that we disperse the light. This means isolating the light from the galaxy, for example by means of a narrow slit. When though few 
photons are involved, one cannot disperse the light without limit. Attempting to obtain more photons by decreasing the dispersion would, on the other hand, cause an observational confusion as light from neighboring galaxies in that part of the sky would also fall on the spectrograph. There is then a complementary inverse relationship between dispersion and brightness which does not permit accurate spectra of faint galaxies to be obtained.

The predictions of various competing models of the universe indicate that the observational horizons complicate the theoretical picture. Moreover, theoretical models present us with their own limitations, what one may call theoretical horizons of knowledge (Kafatos, 1989).

For example, big bang cosmology itself imposes a fundamental limit on the observability of the early universe. What we have is a situation in which direct observation of the early universe based on photons provides information only after a timescale of roughly 100,000 years after the beginning. We can express the age of the universe as a function of redshift $z$. One hundred thousand years after the beginning corresponds to $z$ of 1000 , i.e. when the universe was only $0.1 \%$ of $1 \%$ of its present age. On the other hand, the most distant quasars are seen at a redshift of $\sim 4$ and emitted their light received by us today when the universe was about $10 \%$ of its present age. Radiation can in principle tell us much more about the early universe than matter. However, the opaqueness of the universe prior to $z=1000$ simply does not allow us to trace or confirm big-bang cosmology based on photons. At $z=1000$ we encounter the first theoretical horizon of knowledge about our universe and as long as we are constrained to observe photons, that horizon is impregnable (Kafatos, 1989).

It is unlikely that any other observational means will provide as clear-cut evidence as light about the universe we live in. In principle, primordial neutrinos emitted a few seconds after the beginning of the universe or at a redshift $z \approx 10^{9}$, may one day be observable. Their numbers would be much greater than the supernova neutrinos but their energies billions of times less. Ignoring for the time being the great difficulty in detecting these neutrinos, even if one day we did, they would still not yield any information about the very early universe. The observational horizon of knowledge at $z \approx 10^{9}$ presents, therefore, the ultimate horizon from which we can access direct information about the universe (Kafatos, 1989). Whatever problems of interpretation we are facing today with regards to the background photons will not go away with the neutrinos. The problem of detection and interpretation will only be much worse.

Perhaps considerations from classical cosmological theory can shed more light as to the type of the universe we live in. To test the type of geometry of the universe one studies the Hubble Diagram for distant galaxies, i.e. a diagram of magnitude versus redshift. Quasars can be seen as far away as $z=4$ but galaxies will only begin to be observable at that redshift when the Sapce Telescope starts looking at their spectra in 1990 . Redshifts in the range 5-10 would be particularly important to study since astronomers suspect that at that redshift galaxies began to form more than 13 billion years ago. Unfortunately (Kafatos, 1989) galaxy images begin to blend together at those redshifts and it would be virtually impossible to obtain accurate spectra to study the geometry of the universe. The reason for this 
is because in a general relativistic model of the universe, a finite-size object has an apparent size that decreases until it reaches a minimum (Narlikar, 1983). The curvature of the universe causes the image to decrease for redshifts greater than the minimum value which occurs for $z$ near unity. Obviously, when the apparent size of a galaxy becomes comparable to the mean distance between galaxies, the images blend. For most cosmological models and most "standard" sources this occurs in the approximate redshift range of 1-5. This "galaxy image" theoretical horizon is unfortunately much too close to us. It is in the range where the Space Telescope can obtain spectra of distant galaxies. It is hard to see how the traditional experimental way which revealed the hypothesis of the expanding universe can be carried out to a regime where that hypothesis can be better tested.

As we saw, these horizons which occur inherently in the particular theoretical picture used become worse when the quantum nature of light is considered: Spectra of different sources in the sky would themselves be blended together as one looks at fainter and fainter sources. Eventually, the background from different faint galaxies would dominate the spectrum from a single distant galaxy and reliable spectra could not be obtained.

For all these reasons, in cosmology we encounter horizons of knowledge which prevent us from deciding unequivocally how these tests confirm or reject particular theoretical models (Kafatos, 1989). This is precisely the case where, Bohr insisted, complementarity acquires great importance. One then begins to view the various cosmological models not as rival theories of which one day only one will emerge as the theory of the universe, but as competing complementary constructs. Coupled with the fact that the early universe should be described in quantum terms, one would conclude that these emergent complementary models and the implied underlying wholeness are not an a-priori philosophical preference (Kafatos, 1989) but the very outcome of the observing process for the early universe.

As such, the flatness, horizon and isotropy problems should be tied to the observing process itself rather than as preconditions for theory. As we look at more and more distant galaxies, the universe may be appearing as Euclidean not because of inflation or any other theoretical scheme devised after the facts of specific observational results; rather, because such a universe would naturally emerge as the boundary between complementary constructs. A complementary pair of models, for example, is the open versus the closed universe.

\section{The Quantum Universe}

Modern cosmology faces a fundamental theoretical challenge, how a general relativistic description of the expanding universe rises out of a fundamentally different description of the early cosmos, which must be quantum mechanical in nature. The assumption of classical causality then led to the presumption that subsequent events in larger and larger systems could be known after an observation disclosed the initial conditions in the systems. It was, therefore, reasonable to assume that if the description of the initial conditions in the largest system that can be known, the universe, was sufficiently complete, then we could presume knowledge of all subsequent events in the evolution of the universe. 
Quantum effects in the very early stages of the life of the cosmos were quite large and the most modern of all cosmological theories - a big bang model with inflation - speculates that the universe first came into existence as a result of a quantum transition (Guth and Steinhardt, 1984). The situation will not be resolved until we have at hand a quantized theory of the universe. The subsequent evolution of the universe is described primarily in terms of a theory that we have tended not to recognize as classical in its overall philosophical outlook - the general theory of relativity. And yet it is that theory that will presumably be modified or displaced with a quantum theory of gravity. The seeming universal validity of the general theory has, in other words, contributed to the presumption that the subsequent evolution of the universe after the early initial phase could be understood in terms of the assumption of causality and, therefore, the indeterminacy principle, for all practical purposes, could be ignored. Consequently, most cosmologists tend to view the universe as an idealized closed system that is isolated from the observer and his observing apparatus.

The argument here is simply that the universe is more like a quantum rather than a classical system. It certainly cannot be viewed as a closed system in the sense of being separate from the observer. Quanta in the early universe were tangled together and one expects a similar situation as in the Bell-type experiments (Narlikar, 1983). One would then expect Bell-type correlations to be prevalent in the early universe. For example, in the electron-positron annihilation, the resultant gamma- rays would be polarized perpendicularly to each other. The very physical process of scattering provides then an "observational" or "experimental" choice: The other photon's polarization would not then be presumed to be independent. It would have a well-defined polarization and for the subsequent scattering of the second photon, an isotropic distribution of polarization could not be assumed. We have here a quantum non-local correlation which in principle could stretch across the universe. In other words, quantum effects, however small they might be on the macro level, are pervasive throughout the history of the universe. One cannot in theory at least ever presume a categorical distinction between acts of observation and the observed system, the entire universe. One would then conclude that complementarity will have to be invoked in our efforts to understand the early life of the universe based on observations involving few light quanta. In cosmological observations, the "choice" of whether to record the particle or wave aspect of light from faint sources would have appreciable consequences for our views of the type of the universe we live in.

\section{References}

Barrow, J.D.: 1988, Q. Jl. R. Astr. Soc.,29, 101-117

Barrow, J. D. and Tipler, F. J.: 1986, The Anthropic Cosmological Principle, Oxford Univ. Press, Oxford

Berry, M.: 1976, Principles of Cosmology and Gravitation, Cambridge, Univ. Press, Cambridge

Burns, J. O., Moody, J. W., Brodie, J. P. and Batuski, D. J.: 1988, Ap. J., 355, 542

Dirac, P. A. M.: 1937, Nature, 139, 323

Guth, A. and Steinhardt, P.J.: 1984, Scientific American, June 1984, 116 128 
Kafatos, M.: 1989, in Bell's Theorem, Quantum Theory and Conceptions of the Universe, ed. M. Kafatos, Kluwer Acad. Publ., Dordrecht, p. 195

Narlikar, J.: 1983, Introduction to Cosmology, Jones and Bartlett, New York

Physics Today: Jan. 1989, s. 11

Schramm, D.N.: 1983, Physics Today, April 1983, 27-33 